\title{
Preventing, identifying, and managing medication-related osteonecrosis of the jaw: a practical guide for nurses and other allied healthcare professionals
}

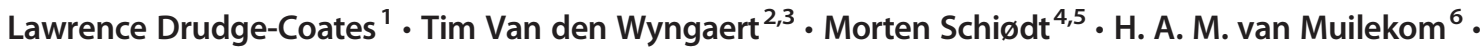 \\ Gaston Demonty ${ }^{7} \cdot$ Sven Otto $^{8}$
}

Received: 20 December 2019 / Accepted: 27 March 2020 / Published online: 19 April 2020

(C) The Author(s) 2020

\begin{abstract}
Background Medication-related osteonecrosis of the jaw (MRONJ) is an infrequent, but potentially serious, adverse event that can occur after exposure to bone-modifying agents (BMAs; e.g., bisphosphonates, denosumab, and antiangiogenic therapies). BMAs are typically used at higher doses to prevent skeletal-related events in cancer patients and at lower doses for osteoporosis/ bone loss. MRONJ can cause significant pain, reduce quality of life, and can be difficult to treat, requiring a multiprofessional approach to care.

Methods We reviewed the literature and guidelines to summarize a practical guide on MRONJ for nurses and other allied healthcare professionals.

Results While there is a risk of MRONJ with BMAs, this should be considered in relation to the benefits of treatment. Nurses and other allied healthcare professionals can play a key role alongside physicians and dentists in assessing MRONJ risk, identifying MRONJ, counseling the patient on the benefit-risk of BMA treatment, preventing MRONJ, and managing the care pathway of these patients. Assessing patients for MRONJ risk factors before starting BMA treatment can guide preventative measures to reduce the risk of MRONJ. Nurses can play a pivotal role in facilitating multiprofessional management of MRONJ by communicating with patients to ensure compliance with preventative measures, and with patients' physicians and dentists to ensure early detection and referral for prompt treatment of MRONJ.

Conclusions This review summarizes current evidence on MRONJ and provides practical guidance for nurses, from before BMA treatment is started through to approaches that can be taken to prevent and manage MRONJ in patients receiving BMAs.
\end{abstract}

Keywords Awareness $\cdot$ Clinical management $\cdot$ Counseling $\cdot$ Jaw necrosis $\cdot$ MRONJ

Electronic supplementary material The online version of this article (https://doi.org/10.1007/s00520-020-05440-x) contains supplementary material, which is available to authorized users.

Lawrence Drudge-Coates

ldrudge-coates@nhs.net

1 Department of Urology, King's College Hospital NHS Foundation Trust, Denmark Hill, London SE5 9RS, UK

2 Department of Nuclear Medicine, Antwerp University Hospital, Edegem, Belgium

3 Faculty of Medicine and Health Sciences, University of Antwerp, Antwerp, Belgium
4 Department of Oral and Maxillofacial Surgery, Rigshospitalet, Copenhagen, Denmark

5 Department of Clinical Epidemiology, Aarhus University Hospital, Aarhus, Denmark

6 Department of Urology, Antoni van Leeuwenhoek HospitalNetherlands Cancer Institute, Amsterdam, Netherlands

7 Medical Development, Amgen (Europe) GmbH, Rotkreuz, Switzerland

8 Department of Oral and Maxillofacial Surgery, Ludwig-Maximilians-University of Munich, Munich, Germany 


\section{Introduction}

Medication-related osteonecrosis of the jaw (MRONJ) is an infrequent, but potentially serious, adverse event associated with bone-modifying agents (BMAs; principally bisphosphonates and denosumab) and antiangiogenic therapies [1-3]. MRONJ has also been reported with tyrosine-kinase inhibitors, mechanistic target of rapamycin inhibitors, $B R A F$ inhibitors, immune checkpoint inhibitors, and cytotoxic chemotherapy [3]. BMAs are typically used at higher doses in patients with cancer to prevent skeletal-related events (SREs) [4], a term that includes the following major complications of bone disease related to tumors: fractures, orthopedic intervention, radiotherapy, and spinal cord compression [5, 6]. At lower doses, BMAs are used to treat osteoporosis and treatment-induced bone loss $[4,7]$.

The risk of MRONJ is greater in patients receiving highdose BMAs (usually for metastatic bone disease) than in those receiving low-dose BMAs (usually for osteoporosis), with a reported incidence of 1-9\% [2] and $0.10 \%$ [8], respectively. Indeed, $90 \%$ of MRONJ cases occur in patients with cancer receiving high-dose BMAs [9].

The pathogenesis of MRONJ has not yet been fully elucidated, but it likely involves several factors, most importantly infection/inflammation, but also impaired bone repair, suppression of osteoclast activity, altered immunity, soft tissue toxicity, and angiogenesis inhibition after exposure to BMAs [10]. The oral microbiome and dental infections are thought to be central to MRONJ development [11, 12].

MRONJ can cause significant pain, reduced quality of life, and can be difficult to treat [1-3], requiring a multiprofessional approach to care. Comprehensive reviews aimed at physicians [13] and dentists [4] have been published. This review is aimed at nurses and other allied healthcare professionals who may encounter patients with or at risk of MRONJ. Early detection of MRONJ can be challenging but is important. Nurses should be vigilant about identifying risks and symptoms, help patients consider the benefit-risk of BMAs, take steps to prevent MRONJ, and facilitate multiprofessional treatment of MRONJ when needed.

\section{Diagnosis of MRONJ and patient identification}

According to the American Association of Oral and Maxillofacial Surgeons (AAOMS), a patient is considered to have MRONJ if they meet all the criteria in Table 1 [14]. An example of exposed MRONJ is shown (Fig. 1a). However, up to one-quarter of patients experiencing MRONJ present with a non-exposed form [15], which should be recognized and treated similarly to exposed MRONJ [16]. Patients presenting with MRONJ with non-exposed bone were not historically formally
Table 1 AAOMS 2014 criteria for diagnosing MRONJ and MRONJ stages [14]

Criteria for diagnosis of MRONJ

1. Current or previous treatment with BMAs or antiangiogenic agents

2. Exposed bone or bone that can be probed through a fistula, situated within or outside the mouth, in the maxillofacial region that has persisted for longer than 8 weeks

3. No history of radiation therapy to the jaws or obvious metastatic disease to the jaws

MRONJ stages

- "At risk" includes patients who have been treated with BMAs but who have no apparent necrotic bone

- Stage 0 includes patients with no clinical evidence of necrotic bone but who have non-specific symptoms or clinical/radiographic findings

- Stage 1 includes patients with exposed and necrotic bone, or fistulae that probe to bone, who are asymptomatic with no evidence of significant adjacent or regional soft tissue inflammation or infection

- Stage 2 includes patients with exposed and necrotic bone, or fistulae that probe to bone, associated with infection, as shown by pain and adjacent or regional soft tissue inflammatory swelling, with or without purulent drainage

- Stage 3 includes patients with exposed and necrotic bone, or fistulae that probe to bone, associated with pain and infection, and at least one of the following: (1) pathologic fracture, (2) an extra-oral fistula, (3) an oral-antral fistula, or (4) radiographic evidence of osteolysis extending to the inferior border of the mandible or the floor of the maxillary sinus

AAOMS, American Association of Oral and Maxillofacial Surgeons; BMA, bone-modifying agent; $M R O N J$, medication-related osteonecrosis of the jaw

diagnosed with the condition $[15,17,18]$. This prompted the AAOMS to include "bone that can be probed through a fistula" as a criterion for MRONJ in 2014 [14]. The AAOMS requirement of at least 8 weeks' observation for MRONJ manifestation may not be necessary [17]. These points are important to keep in mind when assessing for MRONJ.

An example of MRONJ before and after treatment (i.e., healthy tissue) is shown (Fig. 1b, c). MRONJ is staged from "at risk" to stages 0-3 (Table 1) [14]. The signs and symptoms of MRONJ, which can result in difficulties with chewing, eating, and speaking, may include the following: exposed necrotic bone; sinus fistula; signs of infection (e.g., recurrent abscesses, localized bone pain, and bone/gum swelling); bad breath; loose teeth; fracture of the jaw; maxillary sinus pain; and lower lip/chin numbness/heaviness (Fig. 1d) [19]. With the exclusion of exposed necrotic bone, these signs/symptoms may also be caused by dental or other jawbone diseases, which should be excluded before suspecting MRONJ [17]. Jaw pain was reported in over three-quarters of patients who developed MRONJ in an integrated analysis of data from 5723 patients with bone metastases associated with solid tumors/multiple myeloma (MM) in three phase 3 trials of high-dose denosumab or zoledronic acid [20]. Oral infections associated with tooth extractions were reported for nearly one-half of patients with 
Fig 1 Images of MRONJ (a) after tooth extraction of upper first molar in a 55-year-old woman with lung cancer treated with bevacizumab (VEGF inhibitor), (b) another patient with MRONJ before and (c) after treatment (i.e., healthy tissue), and (d) the possible signs and symptoms of MRONJ (modified from [17]) $M R O N J$, medication-related osteonecrosis of the jaw; $V E G F$, vascular endothelial growth factor. Images courtesy of Morten Schiødt (a)

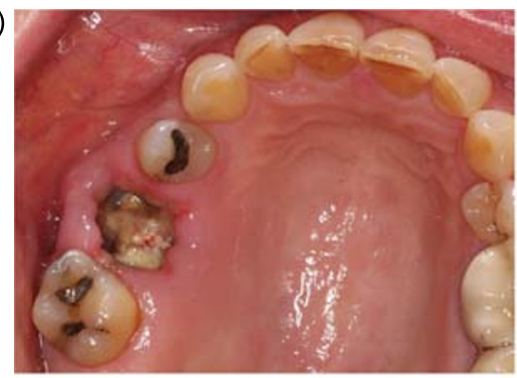

(c)

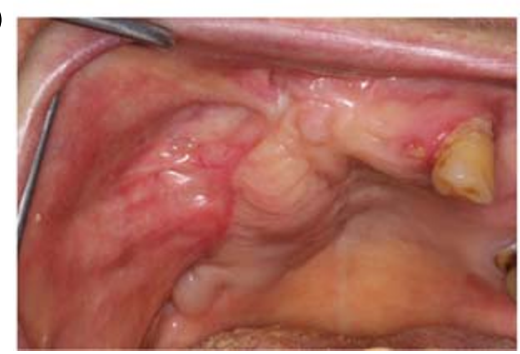

(b)

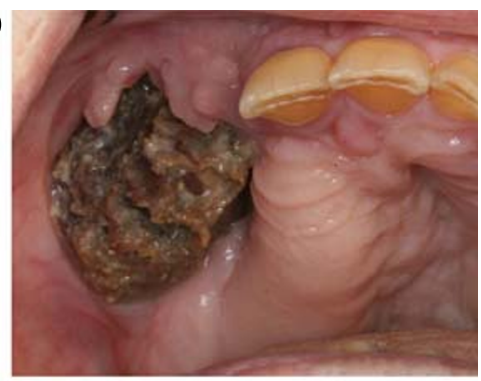

(d) Possible signs and symptoms of MRONJ: Exposed necrotic bone - Sinus/fistula tract* - Recurrent abscesses* - Bone pain

Gingival swelling*

Halitosis*

- Tooth mobility*

- Mandibular fracture

- Maxillary sinus pain

- Lower lip numbness/dysesthesia*

Difficulty swallowing, chewing or speaking *May also be caused by dental or other jawbone diseases, which should be ruled out with clinical and
radiologic investigations before suspecting MRONJ
MRONJ. The most common site of MRONJ was the mandible (less frequently in the maxilla). In a small number of patients $(<5 \%)$, MRONJ was observed in both locations [20].

To enable early detection of MRONJ, nurses can proactively ask "Are there any areas of soreness, numbness or signs of damage in your mouth?" Patients should be prompted to report symptoms such as difficulty with eating and/or speaking [19]. If in doubt, nurses should discuss possible signs and symptoms of MRONJ with the treating physician/dentist, and the patient can be referred to an oral and maxillofacial surgeon or an experienced oral oncology center [21].

\section{Risk factors for MRONJ}

The Multinational Association of Supportive Care in Cancer/ International Society of Oral Oncology/American Society of Clinical Oncology (MASCC/ISOO/ASCO) Clinical Practice Guideline (2019) advises on best practice in the prevention and management of MRONJ in patients with cancer, based on expert opinion and evidence from a comprehensive literature review [2]. This guidance lists risk factors for the MRONJ development; these are summarized in Table 2. Key studies, which elucidated MRONJ risk factors, are discussed in the following sections.

\section{MRONJ risk factors with high-dose BMAs}

Two robust studies evaluated MRONJ risk factors with highdose BMAs: the previously mentioned integrated analysis of data from 5723 patients treated with high-dose denosumab or zoledronic acid in three phase 3 trials [20] and the prospective SWOG0702 trial of approximately 3500 patients with metastatic bone disease receiving zoledronic acid [22].

\section{Table 2 Risk factors for MRONJ [2]}

MASCC/ISOO/ASCO Clinical Practice Guideline significant risk factors for MRONJ

- BMA treatment

- High-dose BMA

- Longer duration of BMA therapy

- Dental extraction and other oral surgical procedures

- Periodontal disease/infection (treatment of infection reduces risk)

- Denture use

- Tobacco use

- Angiogenesis inhibitors

- Diabetes

MASCC/ISOO/ASCO Clinical Practice Guideline factors possibly affecting risk of MRONJ

- Chemotherapy

- Corticosteroids

- Cancer site (for example, breast cancer or multiple myeloma)

- Renal disease

- Erythropoietin therapy

- Hypothyroidism

- Being female

- Being Caucasian

- Older age

$B M A$, bone-modifying agent; MASCC/ISOO/ASCO, Multinational Association of Supportive Care in Cancer/International Society of Oral Oncology/American Society of Clinical Oncology; MRONJ, medicationrelated osteonecrosis of the jaw 


\section{Integrated analysis}

MRONJ incidence was $1.8 \%$ with denosumab and $1.3 \%$ with zoledronic acid (median time on study $\sim 1$ year for both arms) [20]. Tooth extractions and coinciding oral infections occurred in $61.8 \%$ and $48.3 \%$ of patients with MRONJ, respectively. Compared with patients without MRONJ, a greater proportion of those with MRONJ received corticosteroids or antiangiogenic agents (however, only a small number of patients with MRONJ were exposed to antiangiogenic agents). Similar percentages of patients with and without MRONJ had anemia, diabetes, or received chemotherapy. MRONJ incidence increased with time on therapy and thus cumulative BMA exposure.

\section{SWOG0702 trial}

In the SWOG0702 trial, the cumulative incidence of MRONJ at 3 years was $2.8 \%$ overall [22]. Analysis by cancer type demonstrated a higher 3-year risk in MM (4.3 versus $2.9 \%$ for prostate cancer, $2.7 \%$ for lung cancer, and $2.4 \%$ for breast cancer). A zoledronic acid dosing interval of every 3-4 weeks increased the MRONJ risk nearly five times compared with a less frequent dosing regimen (e.g., every 12 weeks). Prior oral surgery nearly doubled the risk, and being a current smoker more than doubled the risk. However, two smaller trials did not show a difference in MRONJ risk between 4 weekly and 12 weekly dosing, illustrating the difficulty in studying the epidemiology of infrequent adverse events in clinical trials that are powered only for efficacy outcomes [23, 24]. The MRONJ risk in SWOG0702 was reduced by approximately one-third in patients with more than the median number of mandibular teeth, and by approximately half in those with more than the median total number of teeth [22]. Tooth loss is often caused by infection (e.g., marginal/apical periodontitis); we hypothesize that the higher incidence of MRONJ in patients with fewer teeth is likely due to infection as an underlying factor. Tooth loss may also be linked to denture use, which approximately doubled the risk of MRONJ [22], likely due to denture-related traumatic ulcers and subsequent exposed bone. Further data from this trial are eagerly awaited.

\section{Other trials examining risk factors associated with high-dose BMAs}

Cumulative BMA dose may be a MRONJ risk factor, as may the type of cancer. Data from two phase 3 studies in breast cancer and prostate cancer showed that the incidence of MRONJ was 1.1 per 100 patient-years during the first year of denosumab treatment, increasing to 4.1 per 100 patientyears in subsequent years [25]. Another phase 3 study in patients with newly diagnosed MM reported a patient-year adjusted incidence of MRONJ of 2.0 per 100 patient-years during the first year of treatment, 5.0 in the second year, and 4.5 per year thereafter [26]. A retrospective study assessed the incidence of, and risk factors associated with, MRONJ in 120 patients with MM treated with bisphosphonates following highdose chemotherapy and autologous stem cell transplantation; 23 (19\%) patients developed MRONJ [27]. Patients with MM therefore deserve particular attention for MRONJ prevention and early detection. The incidence of MRONJ in the retrospective study was associated with rheumatic disease, recent dental manipulations, and elevated C-reactive protein levels (suggesting infection). Previous bisphosphonate exposure, duration of bisphosphonate therapy, cumulative dose of bisphosphonate, and the type of bisphosphonate administered were identified as MRONJ risk factors [27].

\section{MRONJ risk factors with low-dose BMAs}

Identifying risk factors for MRONJ in patients with osteoporosis receiving low-dose BMAs is challenging, as evidence is limited due to the very low frequency of MRONJ. In the FREEDOM extension study, which involved 3591 post-menopausal women with osteoporosis, there were only 12 cases of MRONJ after up to 7 years of low-dose denosumab (plus 3 years of denosumab in the main FREEDOM study) [28]. Nearly half the women had undergone at least one invasive oral procedure, and the incidence of MRONJ was higher in these women compared with those that did not $(0.68$ versus $0.05 \%)$. A systematic review identified 680 cases of MRONJ in patients with osteoporosis from 44 studies [29]. Duration of anti-absorptive drug treatment was found to be a MRONJ risk factor, as was concomitant corticosteroid/immunosuppressive treatment. Dental extractions followed by dentoalveolar surgery were the most frequent events prior to MRONJ, occurring in $48.5 \%$ and $21.1 \%$ of patients, respectively. In these cases, MRONJ was likely triggered by an underlying chronic local infection, which resulted in dental extraction, rather than the procedure [29]. Infection may develop as a consequence of the dental procedure, when bacteria gain access to bone because extraction sockets are left open to heal spontaneously or when wound healing is incomplete [30]. Alternatively, infection may be the reason for the tooth extraction. Indeed, a retrospective study of patients with osteoporosis and/or malignant tumors treated with bisphosphonates undergoing tooth extraction or surgical tooth removal revealed that in $69 \%$ of cases, osteomyelitic or osteonecrotic changes were already present at the time of the extraction [30].

\section{Patient risk assessment}

To assess MRONJ risk in patients before starting a BMA, please see Online resource 1; Supplementary data 1 for questions to ask your patients. If the patient answers "Yes" to any of 
these questions, they have an increased risk of developing MRONJ. Cumulative exposure to BMA is a key risk factor; this includes the dose per treatment, frequency of treatments, and duration of treatment, as well as the potency, but not the route of administration. Studies analyzing administration route as a risk factor tend to be confounded by the fact that denosumab is administrated subcutaneously, regardless of dose, whereas low-dose bisphosphonates tend to be oral and high-dose bisphosphonates tend to be intravenous $[4,31]$. Generally, high-dose BMA prescribed for patients with metastatic cancer is associated with a higher risk of MRONJ compared with lowdose therapy for osteoporosis [17]. In addition to the dose per administration, the intensity of dosing may be up to 12 times more frequent in the setting of bone metastases compared with osteoporosis. Therefore, the dose, frequency, and duration of BMA treatment are included as additional considerations, as is age. Online resource 1; Supplementary data 2 provides a flow chart to assess MRONJ risk. Using this chart, patients receiving high-dose BMAs would automatically be considered to be at elevated risk.

Nurses can play a unique role in translating the benefit-risk ratio of BMAs to patients who may be concerned about the risk of MRONJ. Nurses can do this by educating the patient on the potential advantages of treatment. While this may appear trivial, it is well documented that the patient's perception of risk carries more weight in decision-making than the probability of harm, even if the benefits are clear for oncologic and osteologic indications [32]. This benefit-risk will vary from patient to patient, depending on the individual's risk to develop an SRE (e.g., disease extent, location, and activity), the presence of risk factors for MRONJ, and how a patient perceives having an SRE or developing MRONJ.

In the osteoporosis setting, the FREEDOM study of lowdose denosumab in 4074 post-menopausal women showed an MRONJ incidence rate of 0.05 per 100 patient-years (i.e., 5 cases of MRONJ for every 10,000 years of treatment) [33]. In comparison, the rate of non-vertebral fractures with 3 years of placebo was 2.65 per 100 patient-years (i.e., 265 fractures per 10,000 years) whereas patients treated with 3 years of denosumab had 2.15 fractures per 100 patient-years, and this was significantly reduced to 1.53 per 100 patient-years with a further 4 years of denosumab treatment.

In terms of explaining benefit-risk to the patient, it could be said that "The chance of preventing a SRE using BMA treatment is greater than the risk of developing MRONJ as a result of treatment, and there are steps that can be taken to reduce the risk of MRONJ even further." Statements based on average treatment effects and risks can be derived from clinical trial data and may further assist nurses in communicating the magnitude of benefit-risk to patients, ultimately leading to more informed decisions. For example, a woman with breast cancer that has spread to the bones, who considers the harm that comes from a bone complication to be equal to the harm from MRONJ, could be told that "On average, you are 17 times more likely to derive benefit than harm during the first two years of denosumab treatment, compared with choosing no bone-directed treatment" (Online resource 1; Supplementary data 3).

As patients often give risk more weight than benefit, patient decision aids, such as handouts, may help inform patients on the benefits and harms associated with different therapy options, aiding in shared decision-making [32, 34]. For example, the Scottish Dental Clinical Effectiveness Programme has published dental clinical guidance [35], which includes a section on "Points to Cover During MRONJ Risk Discussion," and provides an illustrated figure for explaining risk to patients (Online resource 1; Supplementary data 4). Tools are available from other therapy areas, for example, The Royal College of Obstetricians and Gynaecologists produced a patient leaflet called "Understanding how risk is discussed in healthcare," which can be found online [36]. Similar aids could be created as valuable tools for oncology nurses when discussing treatment options with patients at risk of MRONJ.

\section{Prevention of MRONJ and implications for nurses and allied healthcare professionals}

In addition to counseling the patient who may have concerns about MRONJ, working with the patient to prevent the condition is a key. Nurses can provide clear explanations, information, and education on MRONJ risks and the preventative steps to reduce this risk. Figure 2 details approaches for preventing and managing MRONJ based on the assessment of MRONJ risk determined by the answers to the questions/flowchart in Online resource 1; Supplementary data $1 / 2$. Nurses can empower patients to be part of the process of preventing MRONJ through following best practice. Ensuring patient compliance with MRONJ preventative measures and any subsequent treatment is vital and requires a level of understanding from the patient.

MRONJ prevention should be started prior to BMA treatment. For low-risk patients, the same recommendations on preventative dental visits and maintaining optimal dental health for the general population apply $[1,2,37]$. For patients at increased risk of MRONJ, the MASCC/ISOO/ASCO Clinical Practice Guideline considers modifiable risk factors to be poor dental care, invasive dental procedures, poorly fitting dentures, high blood sugar, and smoking [2]. Risk reduction strategies to prevent MRONJ before and during denosumab/ bisphosphonate treatment include premedication dental evaluation, maintaining optimal oral hygiene, and having regular dental check-ups $[1,2,14,38]$. An example letter for the patient's dentist, explaining that the patient will be starting a BMA and a dental evaluation is required, as well as information sheets for the dentist, is included in Online resource 1; 
Fig. 2 Approach for preventing and managing MRONJ. BMA, bone-modifying agent; $M R O N J$, medication-related osteonecrosis of the jaw

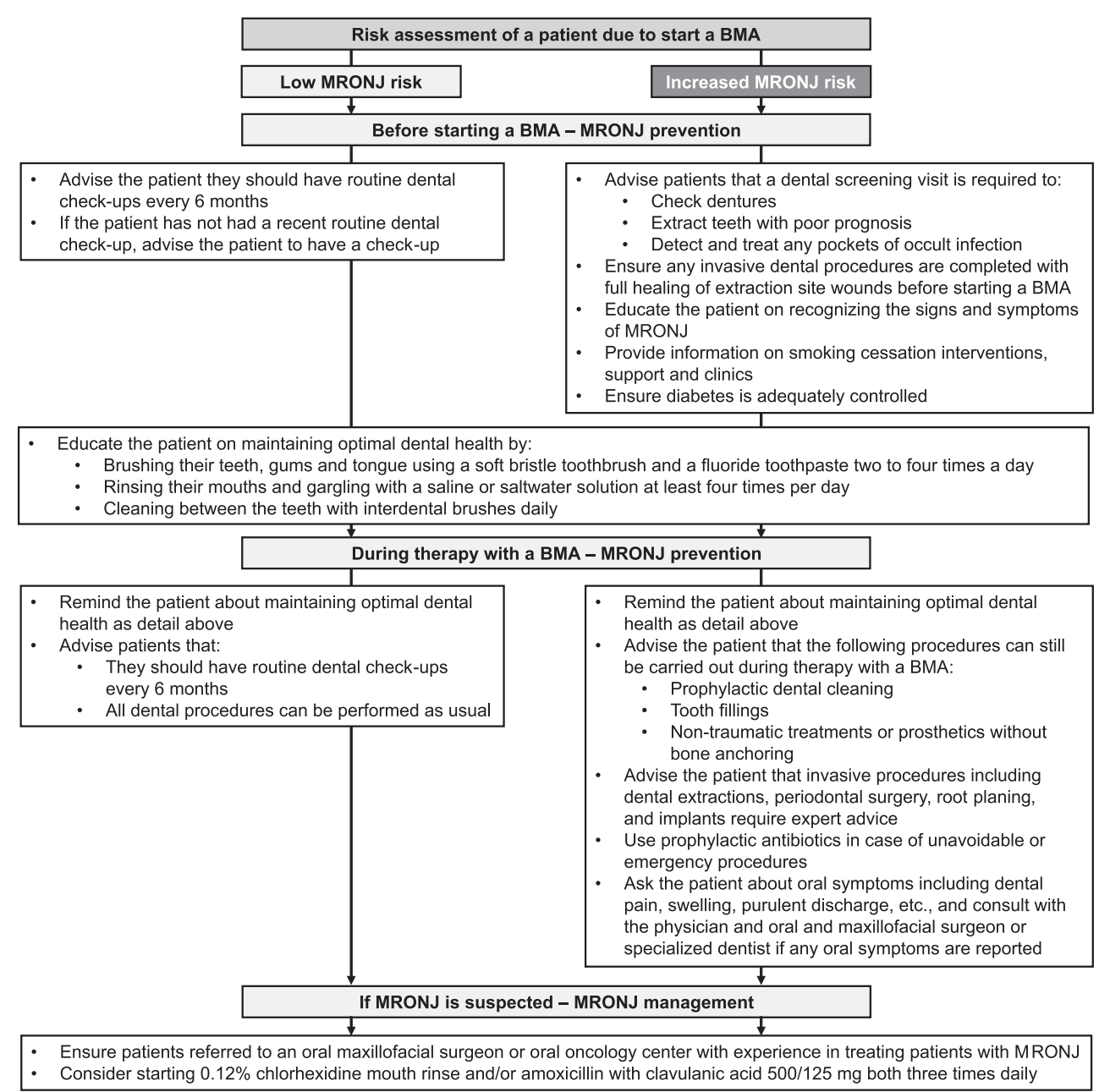

Supplementary data 5. Dental evaluation before starting BMAs has been shown to significantly reduce the MRONJ incidence [39]. A systematic review of risk-reductive dental strategies for MRONJ in six studies of 2332 cancer patients found that preventative dental measures significantly decreased MRONJ incidence by approximately three-quarters versus control groups [40]. Ensuring adequate control of diabetes and providing information on smoking cessation are also important when addressing modifiable risk factors prior to starting a BMA [2].

MRONJ prevention should continue during BMA treatment. It is important to continue to remind patients about preventative dental visits and maintaining optimal dental health. Nurses should stress the importance of monitoring for and reporting symptoms of MRONJ. Patients with a low risk of MRONJ can undergo all dental procedures as usual [14]. Patients with an increased MRONJ risk can still undergo non-invasive dental procedures when necessary but invasive procedures require expert advice [1, 2]. If carried out in accordance with established guidelines, invasive dental procedures, such as a tooth extraction, can be safely performed even in high-risk patients (those receiving BMA in an oncology setting). Low rates of MRONJ have been reported following tooth extraction in a patient population that included those receiving high-dose BMA for malignant disease [41]. Furthermore, it has been speculated that if a tooth extraction is performed to eliminate a local infection, and is performed with preventative measures (antibiotic prophylaxis and plastic wound closure), the procedure may actually decrease the long-term risk of MRONJ [30]. However, no randomized evidence currently supports such a hypothesis.

Barriers to successful MRONJ prevention can include poor patient knowledge or understanding of the recommended preventative strategies [42], which can be addressed by education. In a qualitative study of 23 patients with MRONJ, fear of visiting the dentist, lack of awareness of the importance of oral health, and cost of dental treatment were all identified as potential barriers to MRONJ prevention [42]. Oral health disparities, such as access to oral healthcare, the patient's socioeconomic status, and health literacy, are also noted as barriers in the MASCC/ISOO/ASCO Clinical Practice Guideline [2]. At the healthcare-provider level, oncologists and dentists may not be aware of all BMA-related concerns when treating their patients. In an effort to increase awareness, Patel et al. developed a dental alert card, designed for patients on MRONJ- 
associated therapy, to show their dentist when undergoing assessment/treatment, notifying them of the patient's underlying risk of MRONJ [43]. We propose an example dental alert card that could be adapted in Online resource 1; Supplementary data 6 . Nurses should be mindful of potential barriers and ensure that patients are supported as far as possible in carrying out preventative measures and that appropriate information is exchanged between healthcare providers.

\section{Clinical management of MRONJ}

If MRONJ is suspected, the patient should be referred to either an oral and maxillofacial surgeon, or a specialist oncology center with experience of MRONJ [21]. In the interim, a $0.12 \%$ chlorhexidine mouth rinse and/or amoxicillin with clavulanic acid 500/125 mg three times daily can be prescribed by a dentist or other qualified healthcare professional to treat any related infection (Fig. 2). Patients should be reassured that if MRONJ is diagnosed, the condition is treatable. They should be advised that the oral and maxillofacial surgeon will discuss the treatment options with them, and that this treatment may include surgery.

The AAOMS position paper has assigned treatment strategies according to the stage of MRONJ [14], the goals of which are to prioritize and support continued oncologic treatment and preserve quality of life through patient education and reassurance, and to control infection, progression of bone necrosis, and pain. For the initial treatment of MRONJ, the MASCC/ISOO/ ASCO Clinical Practice Guidelines recommend non-surgical treatments, such as antimicrobial mouth rinses, effective oral hygiene, antibiotics if clinically indicated (i.e., when signs and symptoms of infection are present), and conservative surgical procedures [2], followed by aggressive surgical interventions for refractory cases. Notably, longer exposure time to bisphosphonates and more advanced stages of MRONJ significantly reduce MRONJ healing rates with non-surgical treatments [44]; therefore, it is important to identify and treat MRONJ early. While non-surgical interventions may be appropriate or necessary in certain situations [45-47] (for example, if the aim of therapy is a reduction of symptoms, or if the patient has no symptoms, or has severe symptoms but does not qualify for surgery), mucosal healing is rarely achieved with non-surgical treatments alone [45, 46], and with regard to this outcome, surgery may be more effective [13].

Surgical approaches to treatment include jaw resection, invasive sequestrectomy, and debridement [48]. Surgery may stop disease progression and lead to complete mucosal healing, with success rates of over $90 \%$ reported with certain procedures [45, 47], but the criteria for selecting a patient for surgery are not always agreed upon and require further study. The aim of surgery is to completely remove necrotic bone while avoiding unnecessary removal of healthy bone. Determining the precise margin of necrosis is challenging. Imaging techniques such as bone fluorescence may help to distinguish necrosis from healthy areas, improving the accuracy of surgical procedures $[45,47]$. While surgery allows tissue samples to be extracted for histopathological evaluation and confirmation of MRONJ and/or metastasis, the results almost invariably show necrotic bone without impact on further patient management.

In contrast with the MASCC/ISOO/ASCO recommendations, a 2019 European task force working group on MRONJ concluded that surgical treatment is superior to non-surgical management and also suggested that early surgery for localized disease may be considered to prevent progression [17]. Surgical treatment may be superior to non-surgical interventions in terms of its predictability of duration.

Many other treatment strategies for MRONJ have been proposed. A systematic review of low-intensity laser, hyperbaric oxygen, and platelet-rich plasma concluded that these approaches are well tolerated [49], but there is a lack of evidence from randomized clinical trials [49-51]. Further welldesigned randomized clinical trials are urgently needed to identify the best treatment approach.

\section{A multiprofessional approach to care and organization of the care pathway}

A multiprofessional team, involving nurse practitioners, dentists, physicians, oral oncologists, and oral and maxillofacial surgeons, is important to manage and treat MRONJ [2, 52]. Figure 3 illustrates a multiprofessional approach to preventing and managing MRONJ. The nurse has a role to play in managing the patient's fears and concerns about MRONJ, promoting compliance with preventative measures and treatment, and facilitating communication between the patient, the physician, and the dentist. Lack of cooperation between dentists and physicians may adversely impact the incidence of MRONJ in osteoporosis treatment [53], highlighting an important area in which nurses can assist.

The nurse can also assist in decision-making on further BMA treatment. For example, shared decision-making plays a role when considering "drug holidays." A systematic review of MRONJ treatment found that completely healed sites were significantly more common in patients wh6o had undertaken a BMA "drug-holiday," but this has not been studied in a randomized trial [54]. In addition, there is only limited evidence of benefit for discontinuing BMAs before dentoalveolar procedures, and discontinuing BMAs may increase the risk of SREs and fracture [2]. Therefore, the MASCC/ISOO/ASCO Clinical Practice Guidelines leave this decision to the treating clinicians, recommending that dental specialists are consulted about the risk of MRONJ and oncologists are consulted about potential morbidity as a result of BMA discontinuation [2]. Nurses can play a role in encouraging communication between the physician and dentist, and ensuring that the patient 
Fig. 3 Multiprofessional approach to preventing and managing MRONJ. BMA, bonemodifying agent; $M R O N J$, medication-related osteonecrosis of the jaw

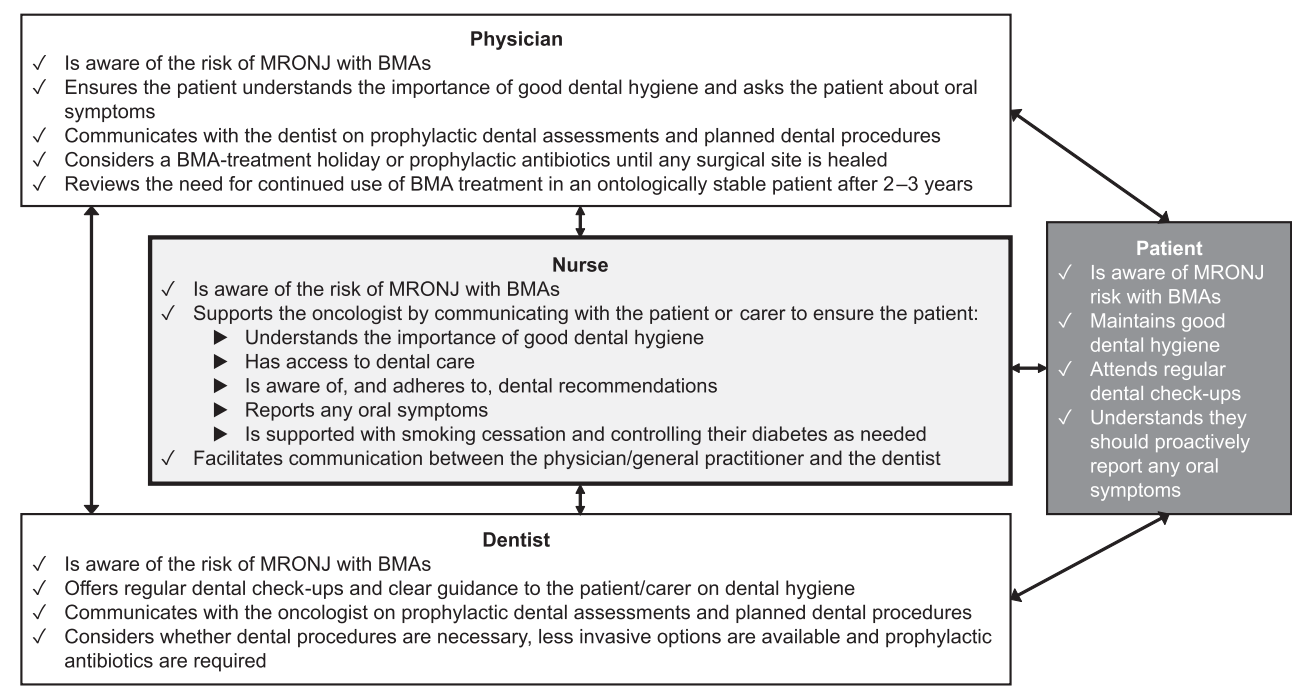

understands the risks and benefits of the approach. Nurses may also be able to help improve the overall organization of the care pathway for patients with suspected MRONJ. For example, nurses might play a role in arranging quick access to an oral and maxillofacial surgeon for a patient who presents with suspicion of MRONJ. They might also promote communication with general practitioners and dentists and act to ensure that all healthcare professionals convey a unified message regarding benefit-risk profiles of BMAs.

Table 3 Key practice points identified by this review article for nurses and healthcare professionals in the identification, prevention, and management of MRONJ

1. To enable detection of MRONJ as early as possible, nurses can proactively ask the patient about the possible signs and symptoms of MRONJ (Fig. 1), and if there is any doubt, nurses should discuss their concerns with the treating physician, dentist, and general practitioner.

2. To assess risk factors for MRONJ, nurses can ask the patient questions based on the known risk factors for MRONJ and determine whether the patient is at low or increased risk of MRONJ (Online resource: Supplementary data $1 / 2$ ).

3. To ensure patient compliance with MRONJ preventative measures and any subsequent treatment, nurses can provide the patient with clear explanations, information, and education on MRONJ risks and the preventative steps that can be taken to reduce this risk (Figs. 2 and 3).

4. To put the risk of MRONJ into context with the patient's other health issues, nurses can educate the patient on how infrequent MRONJ is relative to the benefits of BMA treatment.

5. To ensure appropriate clinical management of MRONJ, nurses can encourage communication between the patient, the physician, and the dentist (Fig. 3).

$B M A$, bone-modifying agent; $M R O N J$, medication-related osteonecrosis of the jaw

\section{Conclusions}

MRONJ occurs in approximately 1-9\% of patients with advanced cancer exposed to high-dose BMAs [2]. Though infrequent, MRONJ is a serious adverse event that can cause significant pain and reduced quality of life, and can be difficult to treat [1-3]. MRONJ is a multiprofessional issue; nurses and other allied healthcare professionals have an important role to play in identifying MRONJ, assessing risk, counseling patients on the benefit-risk of BMAs, and preventing and managing MRONJ. Key practice points highlighted in the review are summarized in Table 3. Healthcare practitioners are urged to enhance their collaboration with one another on a local level to create dedicated care pathways that extend beyond counseling the individual patient.

Author contributions All authors contributed to developing the article concept, critically revising the work, and approving the final version.

Funding information Medical writing support (including development of a draft outline and subsequent drafts in consultation with the authors, assembling tables and figures, collating author comments, copy-editing, fact-checking, and referencing) was provided by Debbie Sherwood BSc (Hon), CMPP, and Sandra Boswell PhD at Aspire Scientific Ltd. (Bollington, UK), and was funded by Amgen (Europe) $\mathrm{GmbH}$ (Rotkreuz, Switzerland).

This article was made open access with the financial support of King's College London, London, UK.

\section{Compliance with ethical standards}

Conflict of interest LD-C has acted as a consultant for Amgen. GD is employed by Amgen and holds stocks in Amgen.

HAMvM has participated in advisory boards for Janssen and Amgen, and received a travel grant from Astellas.

SO has received honoraria from Amgen.

TVdW has received research funding and speaker fees from Amgen. 
MS has acted as a consultant for Amgen, and received research funding from Amgen.

Data sharing statement This manuscript does not include any patient data.

Open Access This article is licensed under a Creative Commons Attribution 4.0 International License, which permits use, sharing, adaptation, distribution and reproduction in any medium or format, as long as you give appropriate credit to the original author(s) and the source, provide a link to the Creative Commons licence, and indicate if changes were made. The images or other third party material in this article are included in the article's Creative Commons licence, unless indicated otherwise in a credit line to the material. If material is not included in the article's Creative Commons licence and your intended use is not permitted by statutory regulation or exceeds the permitted use, you will need to obtain permission directly from the copyright holder. To view a copy of this licence, visit http://creativecommons.org/licenses/by/4.0/.

\section{References}

1. Khan AA, Morrison A, Hanley DA, Felsenberg D, McCauley LK, O’Ryan F, Reid IR, Ruggiero SL, Taguchi A, Tetradis S, Watts NB, Brandi ML, Peters E, Guise T, Eastell R, Cheung AM, Morin SN, Masri B, Cooper C, Morgan SL, Obermayer-Pietsch B, Langdahl BL, Al Dabagh R, Davison KS, Kendler DL, Sandor GK, Josse RG, Bhandari M, El Rabbany M, Pierroz DD, Sulimani R, Saunders DP, Brown JP, Compston J, International Task Force on Osteonecrosis of the Jaw (2015) Diagnosis and management of osteonecrosis of the jaw: a systematic review and international consensus. J Bone Miner Res 30:3-23

2. Yarom N, Shapiro CL, Peterson DE, Van Poznak CH, Bohlke K, Ruggiero SL, Migliorati CA, Khan A, Morrison A, Anderson H, Murphy BA, Alston-Johnson D, Mendes RA, Beadle BM, Jensen SB, Saunders DP (2019) Medication-related osteonecrosis of the jaw: MASCC/ISOO/ASCO clinical practice guideline. J Clin Oncol 37:2270-2290

3. Nicolatou-Galitis O, Kouri M, Papadopoulou E, Vardas E, Galiti D, Epstein JB, Elad S, Campisi G, Tsoukalas N, Bektas-Kayhan K, Tan W, Body JJ, Migliorati C, Lalla RV (2019) Osteonecrosis of the jaw related to non-antiresorptive medications: a systematic review. Support Care Cancer 27:383-394

4. Nicolatou-Galitis O, Schiodt M, Mendes RA, Ripamonti C, Hope S, Drudge-Coates L, Niepel D, Van den Wyngaert T (2019) Medication-related osteonecrosis of the jaw: definition and best practice for prevention, diagnosis, and treatment. Oral Surg Oral Med Oral Pathol Oral Radiol 127:117-135

5. Coleman R, Body JJ, Aapro M, Hadji P, Herrstedt J (2014) Bone health in cancer patients: ESMO clinical practice guidelines. Ann Oncol 25(Suppl 3):iii124-iii137

6. Anderson K, Ismaila N, Flynn PJ, Halabi S, Jagannath S, Ogaily MS, Omel J, Raje N, Roodman GD, Yee GC, Kyle RA (2018) Role of bone-modifying agents in multiple myeloma: American Society of Clinical Oncology clinical practice guideline update. J Clin Oncol 36:812-818

7. Kanis JA, Cooper C, Rizzoli R, Reginster JY, Scientific Advisory Board of the European Society for Clinical Economic Aspects of Osteoporosis (ESCEO) and the Committees of Scientific Advisors and National Societies of the International Osteoporosis Foundation (IOF) (2019) European guidance for the diagnosis and management of osteoporosis in postmenopausal women. Osteoporos Int 30:3-44
8. Lo JC, O’Ryan FS, Gordon NP, Yang J, Hui RL, Martin D, Hutchinson M, Lathon PV, Sanchez G, Silver P, Chandra M, CA MC, Staffa JA, Willy M, Selby JV, Go AS, Predicting Risk of Osteonecrosis of the Jaw with Oral Bisphosphonate Exposure Investigators (2010) Prevalence of osteonecrosis of the jaw in patients with oral bisphosphonate exposure. J Oral Maxillofac Surg 68:243-253

9. Khan A, Morrison A, Cheung A, Hashem W, Compston J (2016) Osteonecrosis of the jaw (ONJ): diagnosis and management in 2015. Osteoporos Int 27:853-859

10. Chang J, Hakam AE, McCauley LK (2018) Current understanding of the pathophysiology of osteonecrosis of the jaw. Curr Osteoporos Rep 16:584-595

11. Otto S, Pautke C, Opelz C, Westphal I, Drosse I, Schwager J, Bauss F, Ehrenfeld M, Schieker M (2010) Osteonecrosis of the jaw: effect of bisphosphonate type, local concentration, and acidic milieu on the pathomechanism. J Oral Maxillofac Surg 68:2837-2845

12. Panya S, Fliefel R, Probst F, Troltzsch M, Ehrenfeld M, Schubert S, Otto S (2017) Role of microbiological culture and polymerase chain reaction (PCR) of actinomyces in medication-related osteonecrosis of the jaw (MRONJ). J Craniomaxillofac Surg 45:357-363

13. Otto S, Pautke C, Van den Wyngaert T, Niepel D, Schiodt M (2018) Medication-related osteonecrosis of the jaw: prevention, diagnosis and management in patients with cancer and bone metastases. Cancer Treat Rev 69:177-187

14. Ruggiero SL, Dodson TB, Fantasia J, Goodday R, Aghaloo T, Mehrotra B, O'Ryan F, American Association of Oral and Maxillofacial Surgeons (2014) American Association of Oral and Maxillofacial Surgeons position paper on medication-related osteonecrosis of the jaw-2014 update. J Oral Maxillofac Surg 72: 1938-1956

15. Fedele S, Bedogni G, Scoletta M, Favia G, Colella G, Agrillo A, Bettini G, Di Fede O, Oteri G, Fusco V, Gabriele M, Ottolenghi L, Valsecchi S, Porter S, Fung PP, Saia G, Campisi G, Bedogni A (2015) Up to a quarter of patients with osteonecrosis of the jaw associated with antiresorptive agents remain undiagnosed. $\mathrm{Br} \mathrm{J}$ Oral Maxillofac Surg 53:13-17

16. Schiodt M, Reibel J, Oturai P, Kofod T (2014) Comparison of nonexposed and exposed bisphosphonate-induced osteonecrosis of the jaws: a retrospective analysis from the Copenhagen cohort and a proposal for an updated classification system. Oral Surg Oral Med Oral Pathol Oral Radiol 117:204-213

17. Schiodt M, Otto S, Fedele S, Bedogni A, Nicolatou-Galitis O, Guggenberger R, Herlofson BB, Ristow O, Kofod T (2019) Workshop of European task force on medication-related osteonecrosis of the jaw-current challenges. Oral Dis 25(7):18151821

18. Migliario M, Mergoni G, Vescovi P, Martino I, Alessio M, Benzi L, Reno F, Fusco V (2017) Osteonecrosis of the jaw (ONJ) in osteoporosis patients: report of delayed diagnosis of a multisite case and commentary about risks coming from a restricted ONJ definition. Dent J 5:E13

19. Ficarra G, Beninati F (2007) Bisphosphonate - related osteonecrosis of the jaws: the point of view of the oral pathologist. Clin Cases Miner Bone Metab 4:53-57

20. Saad F, Brown JE, Van Poznak C, Ibrahim T, Stemmer SM, Stopeck AT, Diel IJ, Takahashi S, Shore N, Henry DH, Barrios CH, Facon T, Senecal F, Fizazi K, Zhou L, Daniels A, Carriere P, Dansey R (2012) Incidence, risk factors, and outcomes of osteonecrosis of the jaw: integrated analysis from three blinded active-controlled phase III trials in cancer patients with bone metastases. Ann Oncol 23:1341-1347

21. Turner B, Ali S, Pati J, Nargund V, Ali E, Cheng L, Wells P (2016) Retrospective audit: does prior assessment by oral and maxillofacial surgeons reduce the risk of osteonecrosis of the jaw in patients receiving 
bone-targeted therapies for metastatic cancers to the skeleton? Part II. Urol Nurs 36:117-133

22. Van Poznak CH, Unger JM, Darke AK, Moinpour C, Bagramian RA, Schubert MM, Hansen LK, Floyd JD, Dakhil SR, Lew DL, Wade JL, Fisch MJ, Henry NL, Hershman DL, Gralow J (2019) Osteonecrosis of the jaw in patients with cancer receiving zoledronic acid for bone metastases: SWOG S0702, NCT00874211. American Society of Clinical Oncology (ASCO) 2019 Annual Meeting

23. Hortobagyi GN, Van Poznak C, Harker WG, Gradishar WJ, Chew H, Dakhil SR, Haley BB, Sauter N, Mohanlal R, Zheng M, Lipton A (2017) Continued treatment effect of zoledronic acid dosing every 12 vs 4 weeks in women with breast cancer metastatic to bone: the OPTIMIZE-2 randomized clinical trial. JAMA Oncol 3:906912

24. Amadori D, Aglietta M, Alessi B, Gianni L, Ibrahim T, Farina G, Gaion F, Bertoldo F, Santini D, Rondena R, Bogani P, Ripamonti CI (2013) Efficacy and safety of 12-weekly versus 4-weekly zoledronic acid for prolonged treatment of patients with bone metastases from breast cancer (ZOOM): a phase 3, open-label, randomised, non-inferiority trial. Lancet Oncol 14:663-670

25. Stopeck AT, Fizazi K, Body JJ, Brown JE, Carducci M, Diel I, Fujiwara Y, Martin M, Paterson A, Tonkin K, Shore N, Sieber P, Kueppers F, Karsh L, Yardley D, Wang H, Maniar T, Arellano J, Braun A (2016) Safety of long-term denosumab therapy: results from the open label extension phase of two phase 3 studies in patients with metastatic breast and prostate cancer. Support Care Cancer 24:447-455

26. Amgen Europe BV (2020) Xgeva (denosumab) summary of product characteristics. https://www.ema.europa.eu/en/documents/ product-information/xgeva-epar-product-information_en.pdf. Accessed 27 Feb 2020

27. Then C, Horauf N, Otto S, Pautke C, von Tresckow E, Rohnisch T, Baumann P, Schmidmaier R, Bumeder I, Oduncu FS (2012) Incidence and risk factors of bisphosphonate-related osteonecrosis of the jaw in multiple myeloma patients having undergone autologous stem cell transplantation. Onkologie 35:658-664

28. Watts NB, Grbic JT, Binkley N, Papapoulos S, Butler PW, Yin X, Tierney A, Wagman RB, McClung M (2019) Invasive oral procedures and events in postmenopausal women with osteoporosis treated with denosumab for up to 10 years. J Clin Endocrinol Metab 104:24432452

29. Aljohani S, Fliefel R, Ihbe J, Kuhnisch J, Ehrenfeld M, Otto S (2017) What is the effect of anti-resorptive drugs (ARDs) on the development of medication-related osteonecrosis of the jaw (MRONJ) in osteoporosis patients: a systematic review. J Craniomaxillofac Surg 45:1493-1502

30. Otto S, Troltzsch M, Jambrovic V, Panya S, Probst F, Ristow O, Ehrenfeld M, Pautke C (2015) Tooth extraction in patients receiving oral or intravenous bisphosphonate administration: a trigger for BRONJ development? J Craniomaxillofac Surg 43:847-854

31. Fleisher KE, Kontio R, Otto S (2016) Antiresorptive drug-related osteonecrosis of the jaw (ARONJ) - a guide to research. AO Foundation, Switzerland: Davos Platz

32. Lewiecki EM (2011) The role of risk communication in the care of osteoporosis. Curr Osteoporos Rep 9:141-148

33. Ferrari S, Butler PW, Kendler DL, Miller PD, Roux C, Wang AT, Huang S, Wagman RB, Lewiecki EM (2019) Further nonvertebral fracture reduction beyond 3 years for up to 10 years of denosumab treatment. J Clin Endocrinol Metab 104:3450-3461

34. Ankolekar A, Vanneste BGL, Bloemen-van Gurp E, van Roermund JG, van Limbergen EJ, van de Beek K, Marcelissen T, Zambon V, Oelke M, Dekker A, Roumen C, Lambin P, Berlanga A, Fijten R
(2019) Development and validation of a patient decision aid for prostate cancer therapy: from paternalistic towards participative shared decision making. BMC Med Inform Decis Mak 19:130

35. Scottish Dental Clinical Effectiveness Programme (2017) Oral health management of patients at risk of medication-related osteonecrosis of the jaw: dental clinical guidance http://www. sdcep.org.uk/wp-content/uploads/2017/04/SDCEP-Oral-HealthManagement-of-Patients-at-Risk-of-MRONJ-Guidance-full.pdf. Accessed 14 Apr 2020

36. The Royal College of Obstetricians and Gynaecologists (2015) Understanding how risk is discussed in healthcare. https://www. rcog.org.uk/globalassets/documents/patients/patient-informationleaflets/pi-understanding-risk.pdf. Accessed 14 Apr 2020

37. European Oral Care in Cancer Group (2017) Oral care guidance and support 2017. http://www.eocc.co.uk/wp-content/uploads/2018/09/ EOCC-English-Guidance.pdf. Accessed 14 Apr 2020

38. Mücke T, Krestan CR, Mitchell DA, Kirschke JS, Wutzl A (2016) Bisphosphonate and medication-related osteonecrosis of the jaw: a review. Semin Musculoskelet Radiol 20:305-314

39. Owosho AA, Liang STY, Sax AZ, Wu K, Yom SK, Huryn JM, Estilo CL (2018) Medication-related osteonecrosis of the jaw: an update on the memorial sloan kettering cancer center experience and the role of premedication dental evaluation in prevention. Oral Surg Oral Med Oral Pathol Oral Radiol 125:440-445

40. Karna H, Gonzalez J, Radia HS, Sedghizadeh PP, Enciso R (2018) Risk-reductive dental strategies for medication related osteonecrosis of the jaw among cancer patients: a systematic review with meta-analyses. Oral Oncol 85:15-23

41. Heufelder MJ, Hendricks J, Remmerbach T, Frerich B, Hemprich A, Wilde F (2014) Principles of oral surgery for prevention of bisphosphonate-related osteonecrosis of the jaw. Oral Surg Oral Med Oral Pathol Oral Radiol 117:e429-e435

42. Sturrock A, Preshaw PM, Hayes C, Wilkes S (2019) Perceptions and attitudes of patients towards medication-related osteonecrosis of the jaw (MRONJ): a qualitative study in England. BMJ Open 9: e024376

43. Patel V, Mansi J, Ghosh S, Kwok J, Burke M, Reilly D, Nizarali N, Sproat C, Chia K (2018) MRONJ risk of adjuvant bisphosphonates in early stage breast cancer. Br Dent J 224:74-79

44. Van den Wyngaert T, Claeys T, Huizing MT, Vermorken JB, Fossion E (2009) Initial experience with conservative treatment in cancer patients with osteonecrosis of the jaw (ONJ) and predictors of outcome. Ann Oncol 20:331-336

45. Otto S, Ristow O, Pache C, Troeltzsch M, Fliefel R, Ehrenfeld M, Pautke C (2016) Fluorescence-guided surgery for the treatment of medication-related osteonecrosis of the jaw: a prospective cohort study. J Craniomaxillofac Surg 44:1073-1080

46. Nicolatou-Galitis O, Papadopoulou E, Sarri T, Boziari P, Karayianni A, Kyrtsonis MC, Repousis P, Barbounis V, Migliorati CA (2011) Osteonecrosis of the jaw in oncology patients treated with bisphosphonates: prospective experience of a dental oncology referral center. Oral Surg Oral Med Oral Pathol Oral Radiol Endod 112:195-202

47. Ristow O, Otto S, Troeltzsch M, Hohlweg-Majert B, Pautke C (2015) Treatment perspectives for medication-related osteonecrosis of the jaw (MRONJ). J Craniomaxillofac Surg 43:290-293

48. Schiodt M, Vadhan-Raj S, Chambers MS, Nicolatou-Galitis O, Politis C, Coropciuc R, Fedele S, Jandial D, Zhang J, Ma H, Saunders DP (2018) A multicenter case registry study on medication-related osteonecrosis of the jaw in patients with advanced cancer. Support Care Cancer 26:1905-1915

49. de Souza TE, de Castro TF, Michellon FC, Passoni ACC, Ortega LJA, Iwaki LCV, da Silva MC (2019) Adjuvant therapies in the 
management of medication-related osteonecrosis of the jaws: systematic review. Head Neck 41:4209-4228

50. Rollason V, Laverriere A, MacDonald LC, Walsh T, Tramer MR, Vogt-Ferrier NB (2016) Interventions for treating bisphosphonaterelated osteonecrosis of the jaw (BRONJ). Cochrane Database Syst Rev 2:CD008455

51. Beth-Tasdogan NH, Mayer B, Hussein H, Zolk O (2017) Interventions for managing medication-related osteonecrosis of the jaw. Cochrane Database Syst Rev 10:CD012432

52. Di Fede O, Panzarella V, Mauceri R, Fusco V, Bedogni A, Lo Muzio L, Sipmo Onj B, Campisi G (2018) The dental management of patients at risk of medication-related osteonecrosis of the jaw: new paradigm of primary prevention. Biomed Res Int 2018: 2684924
53. Taguchi A, Shiraki M, Sugimoto T, Ohta H, Soen S, Japan Osteoporosis S (2016) Lack of cooperation between physicians and dentists during osteoporosis treatment may increase fractures and osteonecrosis of the jaw. Curr Med Res Opin 32:1261-1268

54. Ramaglia L, Guida A, Iorio-Siciliano V, Cuozzo A, Blasi A, Sculean A (2018) Stage-specific therapeutic strategies of medication-related osteonecrosis of the jaws: a systematic review and meta-analysis of the drug suspension protocol. Clin Oral Investig 22:597-615

Publisher's note Springer Nature remains neutral with regard to jurisdictional claims in published maps and institutional affiliations. 